\title{
Medical Applications of the ALPIDE Detector ${ }^{\dagger}$
}

\author{
Monika Varga-Kofarago (D) on Behalf of the Bergen pCT Collaboration \\ HAS Wigner Research Centre for Physics, Konkoly-Thege Miklós út 29-33, H-1121 Budapest, Hungary; \\ varga-kofarago.monika@wigner.mta.hu \\ + This paper is based on the talk at the 18th Zimányi School, Budapest, Hungary, 3-7 December 2018.
}

Received: 18 March 2019; Accepted: 21 May 2019; Published: 24 May 2019

check for
updates

\begin{abstract}
The CERN Large Hadron Collider (LHC) ALICE detector is undergoing a major upgrade in the Second Long Shutdown of the LHC in 2019-2020. During this upgrade, the innermost detector, the Inner Tracking System, will be completely replaced by a new detector which is built from the ALPIDE sensor. In the Bergen proton computer tomography (pCT) collaboration, we decided to apply these sensors for medical applications. They can be used for positioning in hadron therapies due to their good position resolution and radiation tolerance. Dose planning of hadron therapy is calculated currently from photon CT measurements, which results in large uncertainties in the planning and therefore in a necessary enlargement of the treatment area. This uncertainty can be reduced by performing the CT scan using protons. The current contribution shows the development of a sampling calorimeter built from the ALPIDE detector for proton CT measurements and describes the state of the project.
\end{abstract}

Keywords: ALPIDE; silicon sensors; medical applications; proton CT; radiotherapy; computer tomography; cancer; digital sampling calorimeter

\section{Introduction}

The ALICE detector is undergoing a major upgrade in the second long shutdown of the LHC in 2019-2020. During this upgrade, the innermost detector, the inner tracking system (ITS), will be completely replaced by a new detector [1]. This new detector will be equipped with a MAPS-type silicon detector, the ALPIDE (ALICE pixel detector), which was designed specifically for this upgrade [2]. The ALPIDE is produced in a $50 \mu \mathrm{m}$ and a $100 \mu \mathrm{m}$ thick versions and a reverse substrate bias of around $-6 \mathrm{~V}$ can be applied to it to enlarge its depleted region. The ALPIDE and its prototypes have been thoroughly tested in laboratory and test beam measurements. It was required that the sensor has a position resolution around $5 \mu \mathrm{m}$, a detection efficiency above $99 \%$ and a noise occupancy below $10^{-6}$ hits/event/pixel [1]. The ALPIDE fulfills these requirements both before and after irradiating the chips with the radiation doses expected in its lifetime in the ALICE experiment [2].

Due to its low material budget, good position resolution and radiation hardness, the ALPIDE can also be used in other applications where a precise tracking of a high flux of particles is needed. One such application is medical physics, in particular hadron therapy. In these treatments, protons or heavier ions (usually carbon) are used to destroy the DNA of cancer cells. These protons or ions have an energy usually below $200 \mathrm{MeV} / \mathrm{u}$, which is much lower than in the case of the typical particles at the ALICE detector. This means that the ALPIDE has to be tested at these lower energies to see whether the tracking of these particles is possible. The intensity of the beam is also much higher in these medical applications, therefore it needs to be checked maximum how many particles can be distinguished by the ALPIDE in a single frame. This is especially tricky at this low energy, because low-energy particles lose more energy in the silicon, therefore creating more electron-hole pairs, which results in more pixels firing for one passing particle. This makes the distinguishing of particles more difficult at lower energy than at the usual energies of the ALICE detector. 


\section{Computer Tomography with Protons}

The problem of current hadron therapy treatments is that the treatment is planned after acquiring an image of the patient by a photon CT scan. This results in large uncertainties (around 3-4\%) in the determination of the stopping power of protons in front of the tumor [3]. This is due to the fact that the relation of the attenuation coefficients of photons and the stopping power of protons is not linear and not one-to-one as it differs depending on the type and geometrical structure of the tissue [4].

This problem can be solved by using protons for the imaging in the CT measurement instead of photons, therefore the measurement will give directly the stopping power for protons. This would reduce the uncertainty by more than a magnitude to $0.3 \%$ [3]. Such a measurement would use protons with a higher energy than the ones used for the treatment, such that their Bragg peak would fall outside of the patient and in the detector placed behind the patient. The position of the protons has to be determined before entering the patient and after leaving the patient. Behind the patient the energy of the protons has to be measured as well. Before the patient, the position of the protons can be determined by the measurement of the beam position or by a tracking detector with very low material budget (maximum 50-100 $\mu \mathrm{m}$ of silicon). After the patient, the position and the energy measurement can be achieved by a high resolution sampling calorimeter. The concept of such a detector can be seen in Figure 1. If the measurement is done prior to the treatment, it can be used for the planning of the treatment, while if it is done quasi-simultaneously, it can be used for dose verification, dose optimization or patient alignment. We note that patient alignment is not a trivial task, as the unavoidable movement of the patient has to be taken into account during the treatment. This can be done by monitoring the patient with a quasi-simultaneous proton CT measurement without giving relevant additional dose.

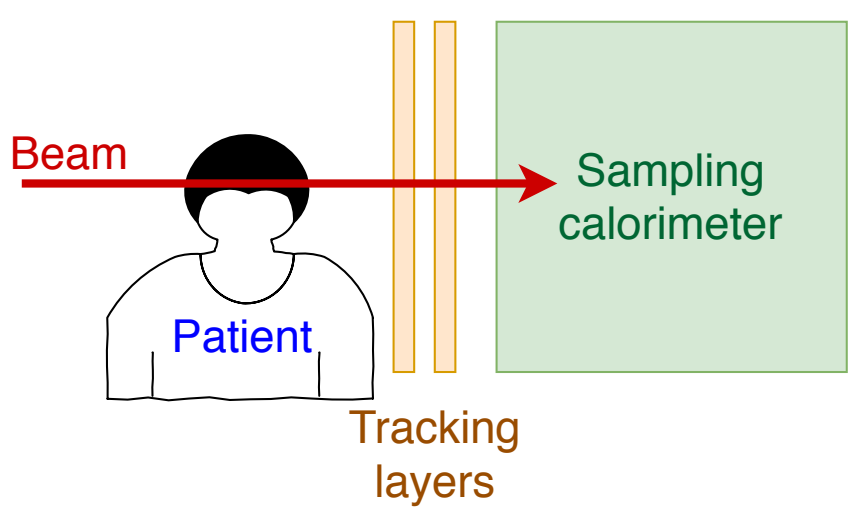

Figure 1. The concept of a proton computer tomography $(\mathrm{CT})$ detector for medical imaging.

\section{The Proposed Calorimeter}

The calorimeter design has the typical sandwich structure well-known for high-energy experimental setups. The active part of the sampling calorimeter will be the ALPIDE sensor. These ALPIDE layers will alternate with aluminum layers which act as energy degraders for the protons. There will be 41 sensitive layers and 41 degrader layers and each aluminum layer will be $3.5 \mathrm{~mm}$ thick. The full front area of the detector will be $27 \mathrm{~cm} \times 15 \mathrm{~cm}$, which is made up of $9 \times 9$ ALPIDE sensors. The proposed calorimeter design can be seen in Figure 2. In the figure, it can also be seen that there will be no aluminum layers between the first few silicon layers. This is done to allow for a more precise tracking of the particles at their entrance to the detector, because this will result in a more precise determination of the position and angle of the incoming particles.

As the ALPIDE was designed to function in the ALICE detector, it is radiation tolerant up to $1.7 \times 10^{13} 1 \mathrm{MeV} \mathrm{n}_{\mathrm{eq}} / \mathrm{cm}^{2}$ non-ionizing dose and up to $2700 \mathrm{krad}$ ionizing radiation. It can be produced in a $50 \mu \mathrm{m}$ and a $100 \mu \mathrm{m}$ thick versions, therefore, if needed, it can be used in the tracker in front of the patient on the beam side as well. 

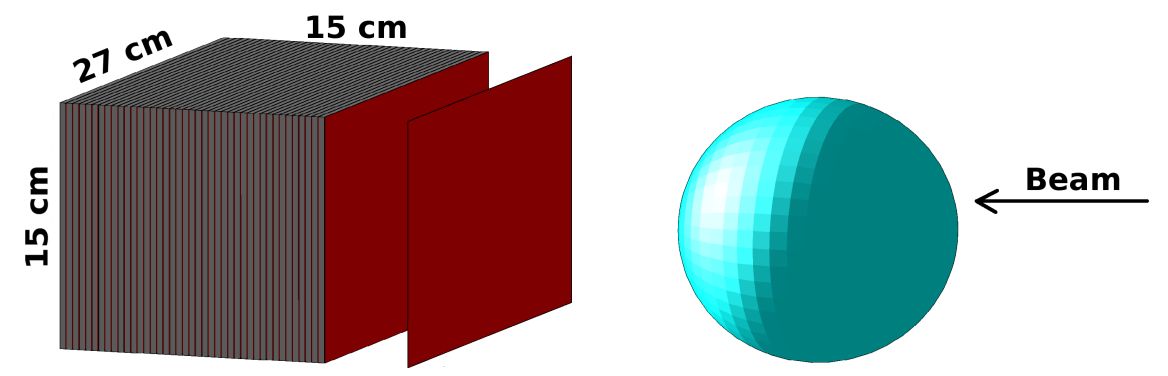

Figure 2. The proposed design of the sampling calorimeter. The red layers are the sensitive layers which alternate with the gray aluminum layers. The blue sphere represents the simplified patient.

\section{Results from the First Prototype Tests}

The first prototype of the calorimeter was not optimized for detecting protons, but for measuring electromagnetic showers. This prototype used MIMOSA23 sensors [5] and used $3.3 \mathrm{~mm}$ tungsten absorbers instead of aluminum as a degrader [6]. It was tested in a proton beam at the KVI-Center for Advanced Radiation Technology in Groningen [7] where the energy was varied from $120 \mathrm{MeV}$ to $188 \mathrm{MeV}$ [8]. The beam energy was changed by introducing an aluminum absorber in the beam line, which introduces a $1.4 \mathrm{MeV}$ energy spread. The beam intensity was set such that one proton per readout frame was delivered to the sensor. The comparison of the results with simulations can be seen in Figure 3 which shows the number of reconstructed protons as a function of their reconstructed range from a $188 \mathrm{MeV}$ proton beam. The protons are reconstructed such that in each layer, the deposited energy is determined from the number of pixels which fire for one passing proton. These deposited energies are then fitted with a Bragg curve, which gives the range of the proton. In both the simulations and the data, the Bragg peak is clearly visible around $230 \mathrm{~mm}$, and the simulation (left panel) describes the test beam data (right panel) well. The lighter green histogram represents nuclear interactions in the detector material, and its structure corresponds to the spacing of the layers. These interactions are not used for the range estimation, as they are not representative for the position of the Bragg peak. Only the dark green points, called accepted tracks, are from reconstructed protons which did not undergo a nuclear interaction and therefore can be used for the estimation of the Bragg peak.
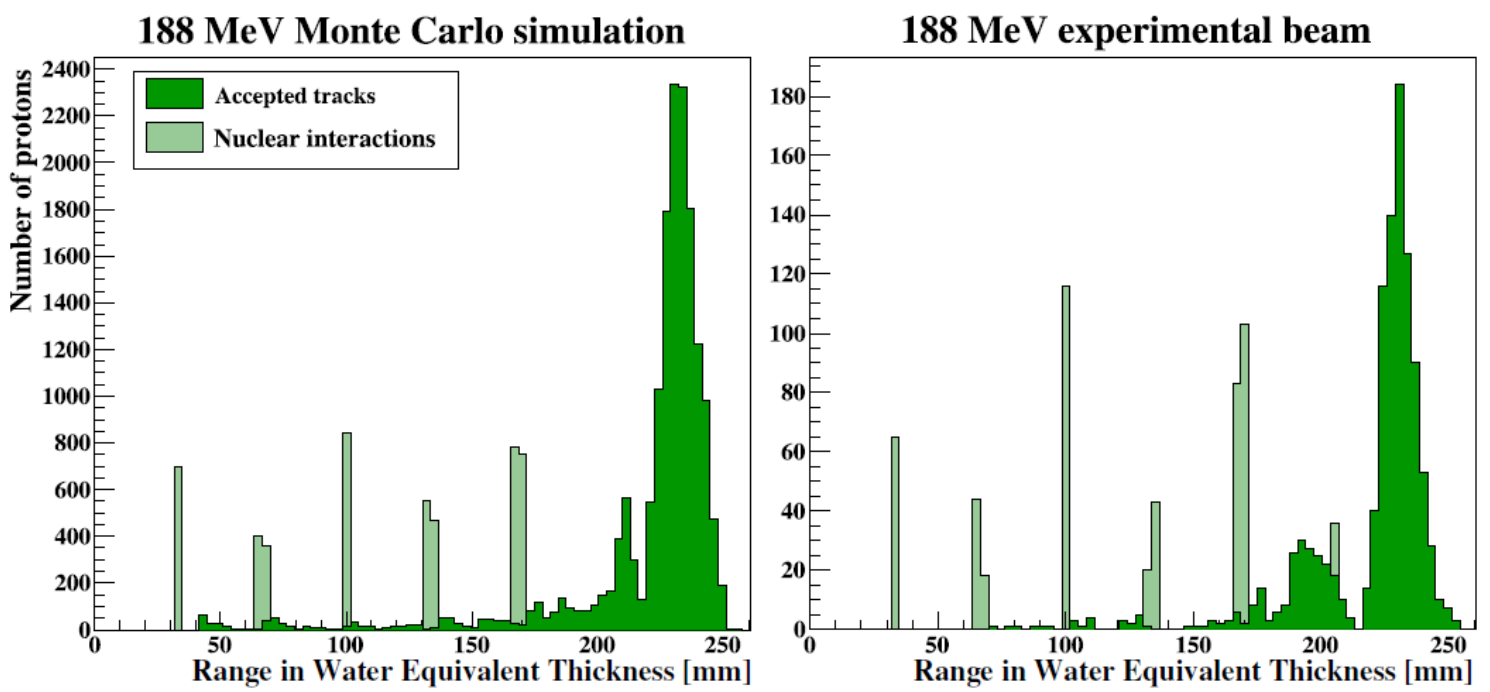

Figure 3. Comparison of the simulations (left panel) and the measured results (right panel) of the prototype with a $188 \mathrm{MeV}$ proton beam at KVI-Center for Advanced Radiation Technology [8]. 
In Figure 4, the reconstructed water equivalent thickness (WET) range of the tracks is shown as a function of the energy of the beam from simulations and the energy scan measurement with a proton beam at KVI-Center for Advanced Radiation Technology. The agreement between data and simulation is good here as well, and the observed linear trend shows that the range is a good measurement of the energy of the incoming protons. An oscillation pattern can be seen in the Monte Carlo simulations which arose form a better estimation of the proton range when the Bragg peak is detected in two sensitive layers and a worse estimation when it is detected in only one. This can be reduced by a further optimization of the absorber thickness.

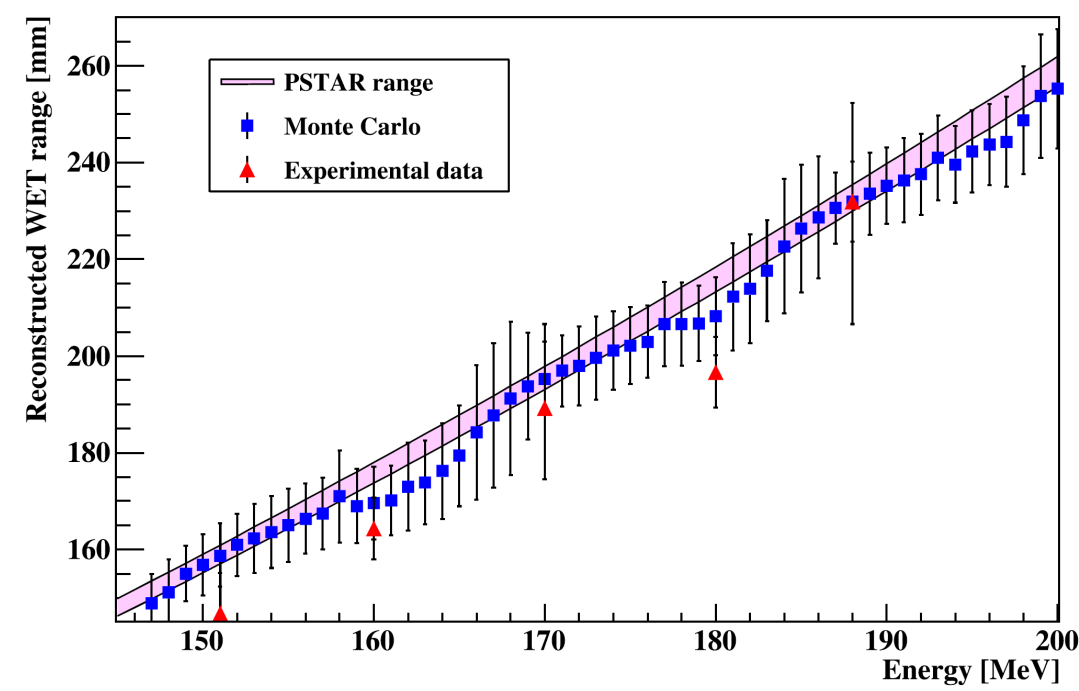

Figure 4. Reconstructed water equivalent thickness range of the protons as a function of the energy of the beam from simulations, from data and from proton stopping power and range (PSTAR) tables that contain numerical integrations of the Bethe equation $[8,9]$.

\section{Conclusions}

A new detector was developed for the CERN LHC ALICE experiment upgrade to replace the current Inner Tracking System after the Second Long Shutdown of the LHC. This detector will be equipped with the ALPIDE sensor, which can also be used for medical applications. A sampling calorimeter of alternating ALPIDE and aluminum layers is proposed as a proton CT detector. With the help of this detector the dose estimation of hadron therapy will become more accurate, therefore it will have less side effects and can be applied closer to critical organs. The first prototype of such a detector is presented here. This was optimized for electromagnetic showers instead of the energy measurement of protons. It shows a good performance, and its performance can be well described by Monte Carlo simulations. A new prototype, which will be optimized for tracking protons, is being built according to the description given in this paper. First one layer of the new prototype, then later the full detector will be tested in test beam measurements with low energy (50-200 MeV) protons and helium or carbon ions.

Funding: This work has been supported by the Hungarian NKFIH/OTKA K 120660 grant and by the Hungarian-Norwegian Bilateral Cooperation Knowledge Exchange Visits to Norway Grants (NKFIH Grant No: HU01-0030-C1).

Conflicts of Interest: The authors declare no conflict of interest. The funders had no role in the design of the study; in the collection, analyses, or interpretation of data; in the writing of the manuscript, or in the decision to publish the results. 


\section{Abbreviations}

The following abbreviations are used in this manuscript:

$\begin{array}{ll}\text { ALICE } & \text { A Large Ion Collider Experiment } \\ \text { ALPIDE } & \text { ALICE pixel detector } \\ \text { CT } & \text { computer tomography } \\ \text { DNA } & \text { deoxyribonucleic acid } \\ \text { LHC } & \text { Large Hadron Collider } \\ \text { MAPS } & \text { Monolithic Active Pixel Sensor } \\ \text { pCT } & \text { proton computer tomography } \\ \text { PSTAR } & \text { proton stopping power and range } \\ \text { WET } & \text { water equivalent thickness }\end{array}$

\section{References}

1. ALICE Collaboration. Technical Design Report for the Upgrade of the ALICE Inner Tracking System. J. Phys. G 2014, 41, 087002. [CrossRef]

2. ALICE Collaboration. The ALPIDE pixel sensor chip for the upgrade of the ALICE Inner Tracking System. Nucl. Instrum. Methods Phys. Res. Sec. A Accel. Spectrom. Detect. Assoc. Equip. 2017, 845, 583-587. [CrossRef]

3. Paganetti, H. Range uncertainties in proton therapy and the role of Monte Carlo simulations. Phys. Med. Biol. 2012, 57, R99-R117. [CrossRef] [PubMed]

4. Schaffner, B.; Pedroni, E. The precision of proton range calculations in proton radiotherapy treatment planning: Experimental verification of the relation between CT-HU and proton stopping power. Phys. Med. Biol. 1998, 43, 1579-1592. [CrossRef]

5. Himmi, A.; Bertolone, G.; Brogna, A.; Dulinski, W.; Colledani, C.; Dorokhov, A.; Hu, C.; Morel, F.; Valin, I. PHASE-1 User Manual; IPHC: Strasbourg, France, 2008.

6. Rocco, E.; FoCal ALICE Group. Highly granular digital electromagnetic Calorimeter with MAPS. Nucl. Part. Phys. Proc. 2016, 273-275, 1090-1095. [CrossRef]

7. KVI-Center for Advanced Radiation Technology. Available online: https://www.rug.nl/kvi-cart/?lang=en (accessed on 24 May 2019)

8. Pettersen, H. A Digital Tracking Calorimeter for Proton Computed Tomography. Ph.D. Thesis, The University of Bergen, Bergen, Norway, 2018.

9. Berger, M.J.; Coursey, J.; Zucker, M.; Chang, J. ESTAR, PSTAR, and ASTAR: Computer Programs for Calculating Stopping-Power and Range Tables for Electrons, Protons, and Helium Ions; National Bureau of Standards: Gaithersburg, MD, USA, 2005. [CrossRef] 